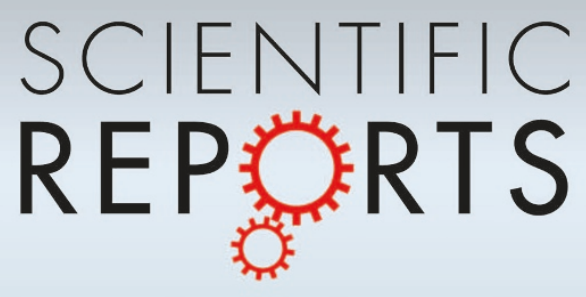

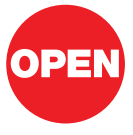

SUBJECT AREAS:

NANOSENSORS

CARBON NANOTUBES AND

FULLERENES

MECHANICAL ENGINEERING

APPLIED PHYSICS

Received

13 March 2013

Accepted

16 April 2013

Published

7 May 2013

Correspondence and requests for materials should be addressed to Q.W. (Q.Wang@ad. umanitoba.ca)

\section{Detection of gas atoms with carbon nanotubes}

\author{
B. Arash \& Q. Wang
}

Department of Mechanical and Manufacturing Engineering, University of Manitoba, Winnipeg, MB, R3T 5V6, Canada.

Owning to their unparalleled sensitivity resolution, nanomechanical resonators have excellent capabilities in design of nano-sensors for gas detection. The current challenge is to develop new designs of the resonators for differentiating distinct gas atoms with a recognizably high sensitivity. In this work, the characteristics of impulse wave propagation in carbon nanotube-based sensors are investigated using molecular dynamics simulations to provide a new method for detection of noble gases. A sensitivity index based on wave velocity shifts in a single-walled carbon nanotube, induced by surrounding gas atoms, is defined to explore the efficiency of the nano-sensor. The simulation results indicate that the nano-sensor is able to differentiate distinct noble gases at the same environmental temperature and pressure. The inertia and the strengthening effects by the gases on wave characteristics of carbon nanotubes are particularly discussed, and a continuum mechanics shell model is developed to interpret the effects.

$T$ he outstanding electrical, mechanical, and thermal properties of carbon nanotubes $(\mathrm{CNTs})^{1-3}$ have made them as one of the most promising materials in development of nanoelectromechanical systems such as nanoscale gigahertz and terahertz devices. The potential of CNTs in nanoscale terahertz devices has opened a wide range of applications in nanotechnology such as atomic transportation, drug delivery, and detection of atoms or molecules ${ }^{4-6}$.

The limitation of gas sensing methods based on the electrical conductance changes of semiconducting $\mathrm{CNTs}^{7,8}$, such as the inability to detect gases with low adsorption energies and distinguish gases in different concentrations, and high sensitivity of nanotube conductivity to changes in environmental conditions, have motivated scientists to build nanoscale gigahertz and terahertz devices for a more precise and robust detection of atoms or molecules $^{9,10}$. The main objective of the nanoscale gigahertz and terahertz devices is to design ultra-sensitive nanosensors based on a vibration or wave propagation analysis. The detection principle by using vibration or wave propagation is to detect the induced recognizable shift in resonant frequencies or the wave velocity in nanosensors owing to attachments of foreign atoms or molecules on surface of the nano-sensors. Static and dynamic mechanical deflections electrically induced to cantilever multi-walled CNTs (MWCNTs) in a transmission electron microscope were developed to measure masses in the pictogram $\left(10^{-12} \mathrm{~g}\right)$ to femtogram $\left(10^{-15} \mathrm{~g}\right)$ mass range $^{11}$. A doubly clamped CNT resonator with a diameter of $3.5 \mathrm{~nm}$ and a length of $300 \mathrm{~nm}$ was experimentally designed as a mass sensor to achieve a detection sensitivity in an order of attogram $\left(10^{-18} \mathrm{~g}\right)$ at room temperature $^{12}$. The experiment enabled the realization of resonance frequencies over $1.3 \mathrm{GHz}$ with nanotube resonators, and furthermore, extremely high resonance frequencies over $10 \mathrm{GHz}$ or even into the terahertz range were expected to be achieved by scaling down CNT-based resonators and selecting suitable coating materials. Ultra-sensitive mass sensing with the resolution of 25 zeptogram $\left(25 \times 10^{-21} \mathrm{~g}\right)$ was experimentally realized by employing a single-walled CNT (SWCNT) resonator with a diameter of $1 \mathrm{~nm}$ and a length of $900 \mathrm{~nm}$ at room temperature ${ }^{13}$. By cooling the nanotube down to $5 \mathrm{~K}$ the resolution was improved to 1.4 zeptogram. The mass sensitivity of nano-resonator sensors was experimentally enhanced to 0.066 zeptogram using a doubly clamped SWCNT resonator with a diameter of $\sim 1 \mathrm{~nm}$ and a length of $150 \mathrm{~nm}$ by which atomic species of Argon (Ar) were weighted $^{14}$. Mass sensing experiments with a resolution of 1.7 yoctograms $\left(1.7 \times 10^{-24} \mathrm{~g}\right)$, which corresponds to the mass of one proton, were reported by using a bridge CNT resonator with a length of $\sim 150 \mathrm{~nm}$ and a diameter of $1.7 \mathrm{~nm}$ as a nano-sensor. The nano-sensors enabled to successfully differentiate a Xenon atom with an atomic mass of 131.29 Da from a naphthalene molecule $(\mathrm{C} 10 \mathrm{H} 8)$ with an atomic mass of $128 \mathrm{Da}$. The experiments assure ultra-sensitive nanotube sensors for detection of atoms and molecules with extremely high accuracy ${ }^{15}$. The potential of SWCNTs in detection of distinct noble gases, i.e., Helium (He), Neon (Ne), Argon (Ar), Krypton (Kr), Xeon (Xe), with a vibration analysis was studied by using molecular dynamic (MD) simulations and a sensitivity index based on frequency shift of the CNTs surrounded by gas atoms was defined ${ }^{16}$. The applicability of SWCNT resonator sensors in detection of distinct genes was investigated through a transverse vibration analysis ${ }^{17}$. Besides 
the vibration analysis of a nano-sensor, the analysis of wave propagation in nano-materials has been also proposed for detection of distinct gas atoms ${ }^{18,19}$. The hypothesis of the detection is that atoms or molecules surrounding a CNT or graphene sensor would induce a shift in the wave velocity, which can be measured technically. The potential of graphene sheets (GSs) as nano-sensors in detection of noble gases through a wave propagation analysis was developed using MD simulations ${ }^{18}$. In above vibration and wave propagation based sensor designs, applying or measuring a harmonic transverse deflection with a frequency in an order of terahertz on CNT or GSsensors is necessary for detection. Although the above sensors have been shown to enable successful detection, two kinds of limitations of the above-proposed sensors hinder their applications. First, applying or measuring such a high-frequency harmonic mechanical excitation on the nano-sensors is not technically sound in practice. For example, applying a high-frequency mechanical vibration in an order of gigahertz or terahertz may be affected by environmental noise signals, which in turn disturb an accurate measurement of frequency shifts in the nano-sensors. On the other hand, the intrinsic ripples in GSs due to their negligible bending rigidity ${ }^{20}$ affect the wave propagation in the sheets, which in turn has influence on accuracy of the detection.

In view of the problems, an introduction and development of a practical CNT sensor for detection of gas atoms are indispensible to achieve a practical detection of gases with higher efficiency by studying propagation of an impulse wave. On one hand, the excitation of an impulse wave is simple and easy to achieve in practice and monitoring of wave propagation due to the impulse wave is also achievable. Hence, the method is technically sound. Moreover, the higher bending rigidity of CNTs compared to graphenes ${ }^{21}$ could prevent any occurrence of ripples in the nanotube sensors to ensure a stable and reliable measurement process.

In this report, the potential of CNTs in design of nano-sensors with an impulse wave propagation analysis in SWCNTs is explored using MD simulations. An index representing the sensitivity based on the wave velocity shifts in the nanotube sensors is defined and examined. It is clearly observed that the proposed nano-sensor is able to successfully differentiate distinct noble gases, i.e., $\mathrm{Ar}, \mathrm{Kr}$, and $\mathrm{Xe}$. Moreover, the inertia effect and the strengthening effect by the gases on wave characteristics of the CNTs were particularly discussed. A continuum mechanics shell model was developed to describe the gas

(a)
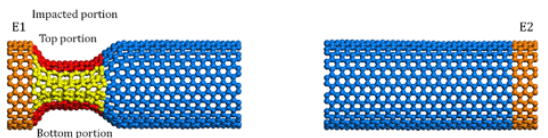

(b)

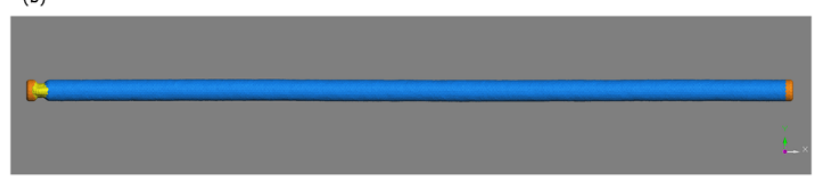

(c)

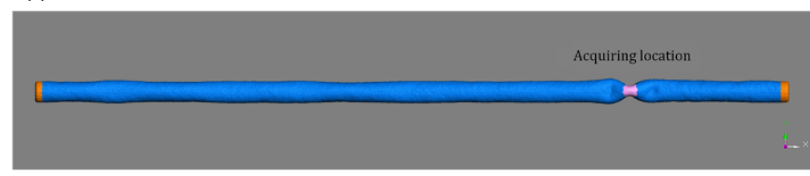

Figure $1 \mid$ Propagation of an impulse wave in a $(22,22) \mathrm{CNT}$ with a length of $\sim 100 \mathrm{~nm}$. Two end of the CNT, i.e., B1 (orange) and B2 (orange), are fixed and the impacted portion (yellow) is subjected to an impact loading as shown in (a) snapshot of the left and right ends of the nanotube at $t=0.05 \mathrm{ps}$. The impact results of an impulse wave in the tube, which propagates from $x_{0}=1.97 \mathrm{~nm}$ as shown in (b) snapshot at $t=0.05 \mathrm{ps}$. The wave reaches the acquiring location (pink) at $x_{a c}=70.11 \mathrm{~nm}$ as illustrated in (c) snapshot at $t_{a c}=19.46 \mathrm{ps}$. detection process. The effects of the some practical considerations, such as the sizes of CNTs, are investigated as well.

\section{Results}

Impulse wave propagation in CNTs. The principle of the detection method on the basis of a wave propagation analysis in a CNT-based gas sensor is to identify a recognizable wave velocity shift in the CNT when it is surrounded with gas atoms with a certain mass density. In order to examine the feasibility of the method, the impulse wave propagation in a pristine $(22,22) \mathrm{CNT}$ with a length of $\sim 100 \mathrm{~nm}$ and fixed on both ends is first investigated. Then the result is compared to that of the same CNT surrounded with Ar gas atoms with a mass density of $1.6 \mathrm{~g} / \mathrm{cc}$ at $298 \mathrm{~K}$.

In simulations, five layers of carbon atoms at two ends, shown as E1 and E2 in Figure 1(a), are fixed by applying restraints to their degrees of freedom. In experiments, the restraints at E1 and E2 can be imposed by techniques of fixing a CNT as an AFM tip and/or forming an intramolecular junction as a restraint point ${ }^{22,23}$. The environmental temperature is set to be $298 \mathrm{~K}$. The CNT is then subjected to an impact loading at the top and bottom of a portion of the CNT shown in red in Figure 1 (a), comprising thirteen layers of carbon atoms indicated as the impacted portion in Figure 1 (a). The impact is assumed to induce an initial velocity of $10 \mathrm{~nm} / \mathrm{ps}$ in the positive and negative directions of the $y$-axis to carbon atoms at the bottom and top of the impacted portion, respectively. As a result, the impact generates an impulse wave along the $\mathrm{x}$-axis in the nanotube which propagates from $x_{0}=1.97 \mathrm{~nm}$ as shown in Figure 1(b) and reaches the acquiring location at $x_{a c}=70.11 \mathrm{~nm}$ at $t_{a c}=19.46 \mathrm{ps}$ as illustrated in Figure 1(c). The process of impulse wave propagation in the pristine $(22,22)$ CNT can be seen in the video attached as Additional Information.

The arrival of the impulse wave at the acquiring location can be detected by means of the transverse deformation of top and bottom atoms, illustrated as T and B in Figure 2, in the direction of the $y$-axis at the acquiring location. In experiments, the motion of the nanotube can be detected using the frequency modulation (FM) mixing technique, which allows transduction of the nanotube motion to a lownoise electrical signal ${ }^{24-26}$. A photonic technique based on the FM method was introduced in Ref. 26, which provided wide and high operating frequencies of up to $1 \mathrm{THz}$. Figure 3 provides a time history of resultant deformations of $\mathrm{T}$ and $\mathrm{B}$ in the direction of the $y$-axis at the acquiring location of $x_{a c}=70.11 \mathrm{~nm}$ at $t_{a c}=19.46 \mathrm{ps}$, pristine $(22,22) \mathrm{CNT}$ subjected to the impulse loading is subsequently (a)

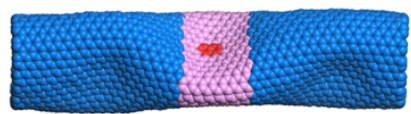

(b)

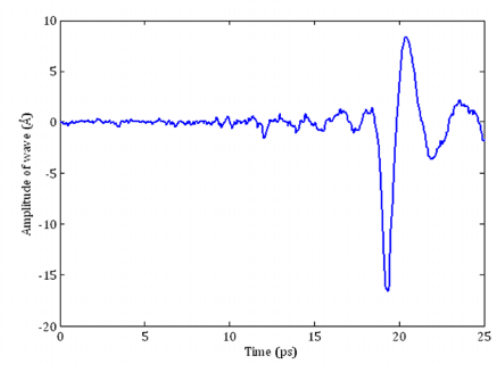

Figure $2 \mid$ Calculation of the wave velocity in the $(22,22)$ CNT. (a) carbon atoms at the top ( $\mathrm{T}$ (red)) and bottom (B (red)) of the CNT at the acquiring location, and (b) time history of the impulse wave in the CNT at the acquiring location at $x_{a c}=70.11 \mathrm{~nm}$. 


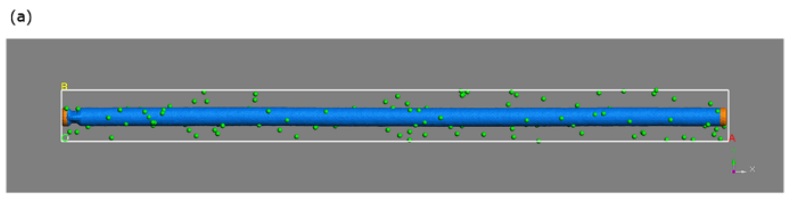

(b)

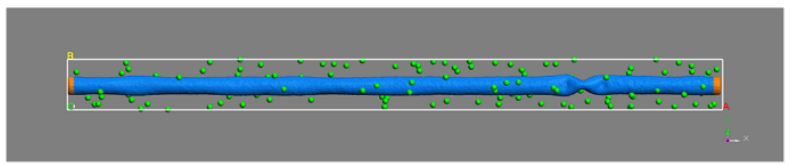

Figure $3 \mid \mathrm{A}(22,22) \mathrm{CNT}$ with a length of $\sim 100 \mathrm{~nm}$ and both fixed ends in a periodic box, filled with 130 Ar atoms, with a size of $102.0 \mathrm{~nm} \times$ $68.0 \mathrm{~nm} \times 68.0 \mathrm{~nm}$ (i.e., the mass density of $1.6 \mathrm{~g} / \mathrm{cc}$ ). (a) at $t=0.05 p s$, (b) the impulse wave reaches acquiring location at $t=19.56 \mathrm{ps}$. determined by calculating the ratio of $\frac{x_{a c}-x_{0}}{t_{a c}}$ that is obtained to be
$c_{0}=3500.95 \mathrm{~m} / \mathrm{s}$.

Following the procedure aforementioned, the impulse wave propagation in the $(22,22) \mathrm{CNT}$ surrounded with Ar gas atoms with a mass density of $1.6 \mathrm{~g} / \mathrm{cc}$ is studied. The corresponding atmospheric pressure for the mass density at the room temperature is $102.3 \mathrm{KPa}$. In the simulation, the CNT is assumed to be immersed in a periodic box of 130 Ar atoms with a size of $102.0 \mathrm{~nm} \times 68.0 \mathrm{~nm} \times 68.0 \mathrm{~nm}$ as illustrated in Figures 3 (a) and (b). The wave velocity in the $(22,22)$ CNT surrounded with Ar atoms with the mass density of $1.6 \mathrm{~g} / \mathrm{cc}$ is obtained to be $c_{g}=3482.41 \mathrm{~m} / \mathrm{s}$. The process of impulse wave propagation in the $(22,22)$ CNT surrounded with Ar gas atoms with a mass density of $1.6 \mathrm{~g} / \mathrm{cc}$ can be seen in the video attached as Additional Information.

To evaluate the sensitivity of the potential CNT-based sensors, a sensitivity index based on the wave velocity shift in the CNT, which is induced by gas atoms, is defined to be $100 \frac{c_{0}-c_{g}}{c_{0}}$, where $c_{0}$ and $c_{g}$ are respectively wave velocities in a pristine CNT and the CNT surrounded with gas atoms. Therefore, the sensitivity of the $(22,22)$ CNT surrounded with Ar gas atoms with the mass density of $1.6 \mathrm{~g} / \mathrm{cc}$ is obtained to be $0.53 \%$. The simulation results show that gas atoms around the CNT induce a decrease in the wave velocity. In addition, the propagation of the impulse wave in the nanotube sensor in absence of any ripples promises a more accurate detection method of gas atoms.

Detection of distinct noble gas atoms. To further explore the applicability of the nanotube sensor subjected to an impact to differentiate distinct gases, the sensitivities of the $(22,22) \mathrm{CNT}$ surrounded with distinct noble gases of $\mathrm{Ar}, \mathrm{Kr}$ and $\mathrm{Xe}$ with various mass densities at the room temperature of $298 \mathrm{~K}$ and pressures ranging from $101.32 \mathrm{KPa}$ to $506.62 \mathrm{KPa}$ are presented in Table 1 . The atomic mass of $\mathrm{Ar}, \mathrm{Kr}$ and Xe are respectively $39.95 \mathrm{~g} / \mathrm{mol}$, $83.8 \mathrm{~g} / \mathrm{mol}$ and $131.29 \mathrm{~g} / \mathrm{mol}$. Thus, at a constant temperature and pressure, the magnitude of the mass density of the three atoms is in the order of Ar, Kr, and Xe. For example, At the room temperature of $298 \mathrm{~K}$ and the pressure of $101.32 \mathrm{KPa}$, the mass densities of $\mathrm{Ar}, \mathrm{Kr}$ and Xe are $1.6 \mathrm{~g} / \mathrm{cc}, 3.7 \mathrm{~g} / \mathrm{cc}$ and $5.9 \mathrm{~g} / \mathrm{cc}$, respectively.

First, the sensitivity of the nanotube sensor in detection of a distinct gas at different densities is studied. From Table 1, the impulse wave velocities in the $(22,22) \mathrm{CNT}$-based sensor surrounded with $\mathrm{Ar}$ gases with the mass densities of $3.3 \mathrm{~g} / \mathrm{cc}, 4.9 \mathrm{~g} / \mathrm{cc}$ and $6.6 \mathrm{~g} / \mathrm{cc}$ are respectively $3464.11 \mathrm{~m} / \mathrm{s}, 3445.97 \mathrm{~m} / \mathrm{s}$ and $3428.02 \mathrm{~m} / \mathrm{s}$, revealing the sensitivity indexes of $1.05,1.57$ and $2.08 \%$. As another example, the impulse wave velocities in the nanotube sensor surrounded with $\mathrm{Kr}$ atoms with mass densities of $6.9 \mathrm{~g} / \mathrm{cc}, 10.3 \mathrm{~g} / \mathrm{cc}$ and $13.9 \mathrm{~g} / \mathrm{cc}$ are respectively $3434.97 \mathrm{~m} / \mathrm{s}, 3410.26 \mathrm{~m} / \mathrm{s}$ and $3353.96 \mathrm{~m} / \mathrm{s}$, showing that the sensitivities increase from $1.88 \%$ to 2.59 and $4.21 \%$, respectively. It is observed that the sensitivity increases monotonically with an increase in the mass density of gas atoms; hence the sensor could successfully estimate mass densities of a distinct gas.

Next, the applicability of the nanotube sensor in detection of distinct gases at the same environmental conditions of temperature and pressure is evaluated. At the room temperature of $298 \mathrm{~K}$ and the pressure of $101.32 \mathrm{KPa}$, the impulse wave velocities in the CNT sensor surrounded with $\mathrm{Ar}, \mathrm{Kr}$ and $\mathrm{Xe}$ gases are respectively $3482.43 \mathrm{~m} / \mathrm{s}, \quad 3464.11 \mathrm{~m} / \mathrm{s}$ and $3445.97 \mathrm{~m} / \mathrm{s}$ as highlighted in Table 1 in blue, showing a decrease in the wave velocity with an increase in the atomic mass of gas atoms. Considering the impulse wave velocity in the pristine $(22,22)$ CNT of $3500.95 \mathrm{~m} / \mathrm{s}$, the sensitivity indexes are respectively found to be $0.53,1.05$ and $1.57 \%$ for $\mathrm{Ar}, \mathrm{Kr}$ and $\mathrm{Xe}$ atoms. As another example, at the same temperate and the pressure of $506.62 \mathrm{KPa}$, where the mass densities of Ar, $\mathrm{Kr}$ and $\mathrm{Xe}$ are respectively $8.2 \mathrm{~g} / \mathrm{cc}, 17.2 \mathrm{~g} / \mathrm{cc}$ and $27.1 \mathrm{~g} / \mathrm{cc}$, the sensitivities respectively increases to $2.34,5.51$ and $6.72 \%$ as highlighted in Table 1 in green. The simulation results justify the potential of the nanotube sensor in detection of distinct gases at the same temperature and pressure even at low concentrations.

After determining the ability of the sensor design in differentiation of distinct gases at the same environmental conditions, the potential of the sensor in detection of gases with close densities is considered. From the simulation results presented in Table 1, the impulse wave velocities in the $(22,22)$ CNT-based sensor surrounded with $\mathrm{Kr}$ and $\mathrm{Xe}$ gas atoms with the mass densities of $10.3 \mathrm{~g} / \mathrm{cc}$ and $10.9 \mathrm{~g} / \mathrm{cc}$ are respectively $3410.26 \mathrm{~m} / \mathrm{s}$ and $3392.68 \mathrm{~m} / \mathrm{s}$ as highlighted in Table 1 in yellow, showing the sensitivity of 2.59 and $3.09 \%$. Therefore, the sensor enables differentiation distinct gases with very close mass densities. In addition, we conduct robust analysis by changing the requiring locations of sensors to investigate the important question from the reviewer. The results show that the sensitivities of the $(22$, 22) CNT sensor surrounded with $\mathrm{Kr}$ atoms and $\mathrm{Xe}$ atoms with same densities in Table 1 are calculated to be $2.5 \% \pm 0.1 \%$ and $3.1 \% \pm$ $0.1 \%$, when the acquiring location, $x_{a c}$, shifts from $70.11 \mathrm{~nm}$ to $80.06 \mathrm{~nm}$. The simulation results reveal the robustness and reliability of the nanotube sensor.

Effect of the diameter of CNTs on the wave velocity. The efficiency of the CNT-based sensor is studied by investigating effect of the diameter of CNT sensors. The impulse wave propagation in (15, 15) and $(22,22)$ CNTs with lengths of $\sim 100 \mathrm{~nm}$ and both fixed ends surrounded with Ar gas atoms with mass densities ranging from 0 (i.e., the pristine CNT) to $18.3 \mathrm{~g} / \mathrm{cc}$ at $298 \mathrm{~K}$ is conducted

Table 1 | Wave propagation in a $(22,22)$ CNT-based sensor with a length of $100 \mathrm{~nm}$ surrounded by noble gas atoms at temperature of $298 \mathrm{~K}$. (Wave velocity in the pristine counterpart of the nanotube sensor is $3500.95 \mathrm{~m} / \mathrm{s}$ )

\begin{tabular}{|lcrcc}
$\begin{array}{l}\text { Type of gas } \\
\text { (atomic mass) }\end{array}$ & $\begin{array}{c}\text { Pressure } \\
(\mathrm{KPa})\end{array}$ & $\begin{array}{c}\text { Density } \\
(\mathrm{g} / \mathrm{cc})\end{array}$ & $\begin{array}{c}\text { Wave velocity } \\
(\mathrm{m} / \mathrm{s})\end{array}$ & $\begin{array}{c}\text { Sensitivity } \\
(\%)\end{array}$ \\
\hline $\operatorname{Ar}$ (39.948) & 101.32 & 1.6 & 3482.43 & 0.53 \\
& 202.65 & 3.3 & 3464.11 & 1.05 \\
& 303.97 & 4.9 & 3445.97 & 1.57 \\
& 405.30 & 6.6 & 3438.02 & 1.80 \\
$\operatorname{Kr}(83.798)$ & 506.62 & 8.2 & 3419.12 & 2.34 \\
& 101.32 & 3.4 & 3460.01 & 1.17 \\
& 202.65 & 6.9 & 3434.97 & 1.88 \\
& 303.97 & 10.3 & 3410.26 & 2.59 \\
$\operatorname{Xe}(131.293)$ & 105.30 & 13.9 & 3353.96 & 4.21 \\
& 506.62 & 17.2 & 3308.06 & 5.51 \\
& 101.32 & 5.3 & 3440.17 & 1.74 \\
& 202.65 & 10.9 & 3392.68 & 3.19 \\
& 303.97 & 16.2 & 3375.28 & 3.59 \\
& 405.30 & 21.8 & 3309.52 & 5.46 \\
& 506.62 & 27.1 & 3265.77 & 6.72 \\
\hline
\end{tabular}


to study the effect. The corresponding atmospheric pressure corresponding to the range of mass density varies from 0 (i.e., the pristine $\mathrm{CNT}$ ) to $1158.90 \mathrm{KPa}$.

Figure 4 provides the results of the wave velocity in the CNTs surrounded with Ar atoms with different mass densities. It is observed in Figure 4 that the wave velocity in the pristine $(15,15)$ CNT with a diameter of $\sim 2 \mathrm{~nm}$ is found to be $3761 \mathrm{~m} / \mathrm{s}$. The wave velocity in the $(15,15)$ CNT surrounded with Ar atoms with a mass density of $3.3 \mathrm{~g} / \mathrm{cc}$ to $6.6 \mathrm{~g} / \mathrm{cc}$ decreases to $3729.06 \mathrm{~m} / \mathrm{s}$ to $3708.05 \mathrm{~m} / \mathrm{s}$. However, additional increase in the mass density of $\mathrm{Ar}$ atoms is unexpectedly found to result in an increase in the wave velocity of the nanotube. The results show that the wave velocity goes up to $3793.52 \mathrm{~m} / \mathrm{s}$ by increasing the mass density of Ar atoms to $18.3 \mathrm{~g} / \mathrm{cc}$. The process of impulse wave propagation in the $(15,15)$ CNT surrounded with Ar atoms with a mass density of $18.3 \mathrm{~g} / \mathrm{cc}$ can be seen in the video attached as Additional Information. The observations demonstrate that there is a turning point at the mass density of $6.6 \mathrm{~g} / \mathrm{cc}$. Before the density, the wave velocity decreases with an increase in the mass density of gas atoms, while after the mass density the wave velocity increases in contrast. The non-monotonic behavior of the wave velocity versus the mass density of surrounding gas atoms found in the $(15,15)$ nanoube, which is not desirable in design of a nano-sensor, needs to be prevented in real applications. A remedy for the non-monotonic wave solutin in CNTs is to increase the diamter of the CNT-sensor. Now, the effect of diameter of CNTs on the wave velocity is investigated. Figure 4 indicates that the wave velocity in the $(22,22)$ CNT with a diameter of $\sim 3 \mathrm{~nm}$ surrounded with Ar atoms with a mass density ranging from 0 to $18.3 \mathrm{~g} / \mathrm{cc}$. From Figure 4 , the wave velocity in the pristine $(22,22)$ CNT is $3500.95 \mathrm{~m} / \mathrm{s}$ that respectively decreases to $3464.08 \mathrm{~m} / \mathrm{s}, 3428.02 \mathrm{~m} / \mathrm{s}$ and $3392.68 \mathrm{~m} / \mathrm{s}$ when the tube is surrounded with $\mathrm{Ar}$ atoms with mass densities of $3.3 \mathrm{~g} / \mathrm{cc}, 6.6 \mathrm{~g} / \mathrm{cc}$ and $18.3 \mathrm{~g} / \mathrm{cc}$. Hence, the wave velocity becomes a monotonically decreasing variaion versus the mass density of gas atoms surrounding the nanotubes with a larger diameter. The monotonic behavior of the wave velocity versus the mass density of surrounding gas atoms, attained by increaing the diameter of a nanotube from $2 \mathrm{~nm}$ to $3 \mathrm{~nm}$, guarantees the effectiveness and reliability of the nano-sensor.

From the simulations, we find that the propagation of an impulse wave in a CNT is affected by two opposite phenomena: (1) the inertia effect, and (2) the strengthening effect. The inertia effect, caused by the vdW interactions between carbon atoms of the CNT and surrounding gas atoms, dissipates the energy of the impulse wave. The effect causes a resistance to the motion of the wave in the CNT and induces a reduction in the wave velocity. Unlike the inertia effect that reduces the wave velocity, gas atoms around the nanotube at the mass densities greater than $6.6 \mathrm{~g} / \mathrm{cc}$ play a role of a surface constrainer on the tube. The surface constrainer on the nanotube makes the tube stiffer, and hence speeds up the wave in longitudinal direction of the nanotube. Herein, the effect is called the strengthening effect.

For nanotubes with a diameter of $2 \mathrm{~nm}$ such as the $(15,15) \mathrm{CNT}$, two regions can be recognized: (1) for the mass densities of gas atoms smaller than $6.6 \mathrm{~g} / \mathrm{cc}$, the inertia effect is dominant, and hence the wave velocity displays a decreasing variation versus the mass density of surrounding gas atoms; (2) for mass densities greater than $6.6 \mathrm{~g} / \mathrm{cc}$ the strengthening effect against is dominant, and hence the wave velocity is found to increase with an increase in the mass densities of gas atoms. Therefore, a turning point is detectable for the wave velocity in the $(15,15) \mathrm{CNT}$ at the mass density of Ar atoms of $6.6 \mathrm{~g} / \mathrm{cc}$ in Figure 4. However, for nanotubes with a diameter of $3 \mathrm{~nm}$ or larger such as the $(22,22) \mathrm{CNT}$, the inertia effect is always dominant so that the wave velocity would be a monotonically decreasing function of the mass density of surrounding gas atoms.

The complicated phenomena of the CNT diameter effect on the wave propagation can be interpreted by using a continuum mechanics model. In the continuum model, the CNT is modeled with a cylindrical elastic shell and the interactions between the nanotube and gas atoms are also simulated with surface stress effects ${ }^{27}$ as shown in Figures 5(a) and (b). Consider a cylindrical elastic shell of radius $r$, thickness $h$, bending rigidity $D$, Young's modulus $E$ and density $\rho$ which are used to model a CNT. If $x$ and $\theta$ respectively represent the longitudinal and circumferential coordinates the governing equation

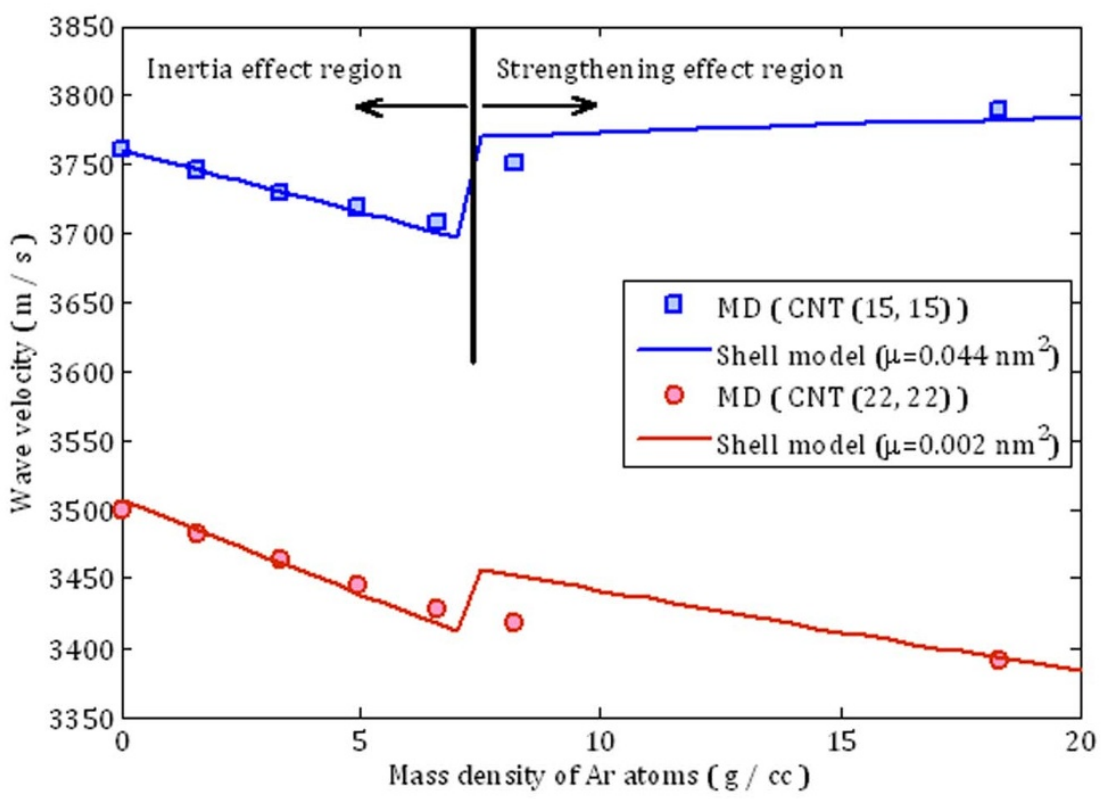

Figure $4 \mid$ Wave velocity in $(15,15)$ and $(22,22)$ CNTs with lengths of $\sim 100 \mathrm{~nm}$ surrounded by Ar atoms versus the mass density of the gas atoms. Before the mass density of $6.6 \mathrm{~g} / \mathrm{cc}$, the wave velocity in the $(15,15)$ CNT decreases since the inertia effect of gas atoms on the nanotube is dominant, while after that the mass density the strengthening effect is governing and the wave velocity increases. In contrast, the wave velocity in the (22, 22) CNT is monotonically decreasing versus the mass density of gas atoms. The wave velocity in the $(15,15)$ CNT is monotonically decreasing when the mass density of gas atoms is lower than $6.6 \mathrm{~g} / \mathrm{cc}$ since two opposite effects of the inertia and the strengthening effects. 
based on the Donnell shell theory including surface stress ${ }^{27}$ and the small-scale ${ }^{28}$ effects in term of the radial displacement $W(x, \theta)$ is given as

$$
\begin{aligned}
\left(D+D^{s}\right) \nabla^{8} W= & 2 \tau^{s}\left(1-\mu \nabla^{2}\right) \nabla^{6} W+\left(\rho h+\rho^{s}\right)\left(1-\mu \nabla^{2}\right) \frac{\partial^{2}}{\partial t^{2}} \nabla^{4} W \\
& +\frac{v h^{2} \rho^{s}}{6(1-v)}\left(1-\mu \nabla^{2}\right) \frac{\partial^{2}}{\partial t^{2}} \nabla^{6} W-\frac{E h}{r^{2}} \frac{\partial^{4} W}{\partial x^{4}}
\end{aligned}
$$

where $D^{s}$ and $\rho^{s}$ are respectively the bending rigidity and the mass density of the surface, $\tau^{s}$ is the residual surface stress under unstrained conditions, and $\mu$ is the small-scale parameter.

Surface effects become prominent when the surface-to-bulk ratio of nanostructures increases ${ }^{29}$. The reason is that the nature of atoms on the surface is different from that in the interior (bulk). Thus, the surface effects are considerable in determining the physical properties of CNTs, when gas atoms adhere to their surface.

The wave propagation solution for CNTs can be expressed as

$$
W=\bar{W} e^{i(k x+m \theta-\omega t)},
$$

where $\bar{W}$ is amplitude of the wave motion, $k=2 \pi / \lambda$ is the wavenumber in the longitudinal direction, $m$ is the wavenumber in the circumferential direction, $\omega=2 \pi c / \lambda$ is the frequency of the wave motion, $c$ is the wave velocity, and $\lambda$ is the wave length.

Assuming $\tau^{s}=0$, substituting Eq. (2) into Eq. (1) gives the wave velocity as

$$
c^{2}=\frac{\left(D+D^{s}\right) \beta^{4}+\frac{E h}{r^{2}} k^{4}}{\left[\left(\rho h+\rho^{s}\right)-\frac{v h^{2} \rho^{s}}{6(1-v)} \beta\right] \beta^{2}(1+\mu \beta) k}, \beta=k^{2}+\frac{m^{2}}{r^{2}} .
$$

From Figures 6 (a) and (b), the wavelength and the circumferential wavenumber are measured to be $\lambda=4 \mathrm{~nm}$ and $m=4$ for a $(15,15)$ CNT, respectively.

The following properties are taken for CNTs and the surface of gas atoms around nanotubes.

$$
\begin{aligned}
& D=0.85 \mathrm{ev}, E=1 \mathrm{TPa}, v=0.27, \rho=2300 \mathrm{~kg} / \mathrm{m}^{3}, \\
& h=0.34 \mathrm{~nm}, \rho^{s}=2 \rho_{g} h \\
& D^{s}= \begin{cases}0, & \rho_{g} \leq 6.6 \mathrm{~g} / \mathrm{cc} \\
0.0017 \rho_{g} \mathrm{ev}, & \rho_{g}>6.6 \mathrm{~g} / \mathrm{cc}\end{cases}
\end{aligned}
$$

where $\rho_{g}$ is the mass density of surrounding gas atoms. From MD simulations presented in Figure 4, the strengthening effect of gas atoms on nanotubes is negligible for $\rho_{g} \leq 6.6 \mathrm{~g} / \mathrm{cc}$, hence $D^{s}$ is set to be zero for this region. Also, $D^{s}=0.0017 \rho_{g} e v$ is taken to be a linear function of the mass density of gas atoms for $\rho_{g}>6.6 \mathrm{~g} / \mathrm{cc}$ to include the strengthening effect. The value is obtained through a fitting (a)

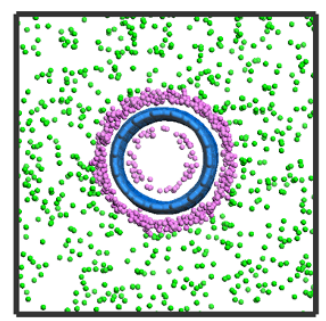

(a)

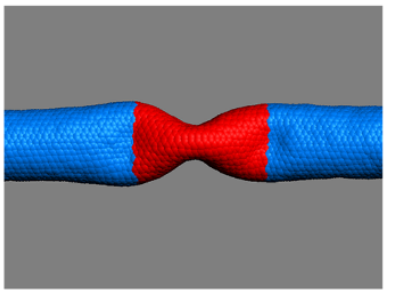

(b)

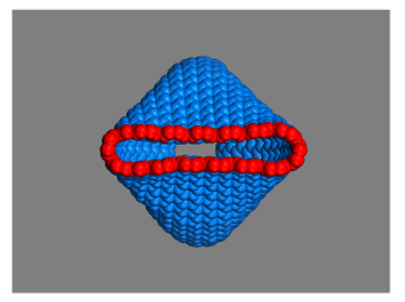

Figure 6 Snapshots of propagation of the wave from: (a) side view for measuring the wave length $(\lambda)$, (b) cross section of the wave for obtaining the circumferential wavenumber $(m)$.

process with results of MD simulations. In addition, the small-scale parameter is respectively set to be $\mu=0.044 \mathrm{~nm}^{2}$ and $\mu=0.002 \mathrm{~nm}^{2}$ for the $(15,15)$ and $(22,22)$ CNTs through a verification process with results obtained from $\mathrm{MD}$ simulations.

Finally, results obtained from the continuum shell model presented in Eq. (3) obviously confirm that there is a turning point for the wave velocity in the $(15,15)$ CNT with a diameter of $2 \mathrm{~nm}$ at the mass density of Ar atoms of $6.6 \mathrm{~g} / \mathrm{cc}$ as shown in Figure 4. For example, the wave velocity in the $(15,15)$ CNT decreases from $3760.53 \mathrm{~m} / \mathrm{s}$ to $3701.57 \mathrm{~m} / \mathrm{s}$ with an increase in the mass density of surrounding Ar atoms from 0 to $6.6 \mathrm{~g} / \mathrm{cc}$. Also, results obtained from the shell model show that the wave velocity would be a monotonically decreasing function of the mass density of surrounding Ar atoms by increasing the diameter of the nanotube to $3 \mathrm{~nm}$. For instance, the wave velocity in the $(22,22)$ CNT predicted with Eq. (3) decreases from $3507.81 \mathrm{~m} / \mathrm{s}$ to $3394.64 \mathrm{~m} / \mathrm{s}$ with an increase in the mass density of surrounding Ar atoms from 0 to $18.3 \mathrm{~g} / \mathrm{cc}$.

\section{Discussion}

The potential of CNTs as nano-sensors in detection of noble gases is explored with an impulse wave propagation analysis. The wave propagation in the nanotube sensors surrounded with different noble gas atoms is simulated by MD simulations. It was demonstrated that a $(22,22)$ CNT with a diameter of $3 \mathrm{~nm}$ and a length of $100 \mathrm{~nm}$ as a nano-sensor could successfully differentiate distinct noble gases, i.e., of $\mathrm{Ar}, \mathrm{Ne}$ and $\mathrm{Xe}$, at the same environmental conditions of temperature and pressure. The sensitivity of the nano-sensor increases with an increase in the mass density of gas atoms. In addition, the nanosensor enables detection of different types of noble gases with close mass densities. The inertia and the strengthening effects of gases on the characteristics of impulse wave propagation in nanotubes are studied, and a continuum mechanics shell model is presented to physically describe the effects. The effect of size of nanotubes surrounded with gas atoms on the impulse wave propagation is investigated as well. The sensitivity analysis in the research shows the great potential of the method for nano-sensors.

\section{Methods}

The interaction potential is modeled by the universal force field (UFF) ${ }^{29}$, which is a purely diagonal and harmonic force field. In the UFF, The potential energy of an arbitrary geometry of a molecule is provided as a superposition of various two-body, three-body, and four-body interactions. The total potential energy, $E$, is expressed as follows ${ }^{30}$ :

$$
E=E_{R}+E_{\theta}+E_{\varphi}+E_{\omega}+E_{v d W}
$$

where $E_{R}, E_{\theta}, E_{\varphi}$ and $E_{\omega}$ are valence terms of bond stretching, bond angle bending, dihedral angle torsion, and inversion energies, respectively. $\mathrm{E}_{v d W}$ is the van der Waals $(\mathrm{vdW})$ energy represented by a sum of repulsive and attractive Lennard-Jones terms ${ }^{31}$. Molecular dynamics simulations are initialized with a geometry optimization process to minimize the total energy of the system (i.e., a CNT surrounded with gas atoms illustrated in Figures 3(a) and (b)) by using the conjugate-gradient method ${ }^{32}$. Once the minimization process is completed, the system is then allowed to equilibrate over the constant volume and constant temperature (NVT) ensemble at the room temperature of $298 \mathrm{~K}$ for $0.5 \mathrm{~ns}$. The simulation time step is set to be $1 \mathrm{fs}$ in the NVT simulation, and the Andersen feedback thermostat ${ }^{33}$ is used for the system
Figure 5 | Modeling a CNT surrounded with gas atoms using an elastic shell model. The CNT is illustrated in blue and surrounding atoms inside and outside of the tube are shown in violet: (a) top view of a $(15,15) \mathrm{CNT}$ surrounded with Ar atoms with a mass density of $6.6 \mathrm{~g} / \mathrm{cc}$, and (b) the equivalent shell model from top view. 
temperature conversion. A NVE (i.e., constant volume and constant energy dynamics) simulation process of $25 \mathrm{ps}$ is then followed to model the propagation of an impulse wave in the CNT. The wave is induced by applying an impact loading at the top and bottom of the impacted portion of the CNT as shown in red in Figure 1 (a). The Verlet velocity algorithm is adopted in the NVE simulation to integrate the motion of equations for the whole system, and the time step in the NVE simulation is chosen to be $0.05 \mathrm{fs}$. The trajectories of atoms at the acquiring location of the CNT, shown in Figure 1(c), are recorded to detect reaching the wave to the acquiring location. The MD simulations have been performed with the Forcite module of Materials Studio software package.

1. Iijima, S. Helical Microtubules of Graphitic Carbon. Nature 354, 56-58 (1991)

2. Wilder, J. W. G., Venema, L. C., Rinzler, A. G., Smalley, R. E. \& Dekker, C. Electronic Structure of Atomically Resolved Carbon Nanotubes. Nature 391, 5962 (1998).

3. Ball, P. Roll up for the Revolution. Nature 414, 142-144 (2001).

4. Wang, Q. Atomic Transportation via Carbon Nanotubes. Nano Lett. 9, 245-249 (2009).

5. Wu, N., Wang, Q., Arash, B. Ejection of DNA molecules from carbon nanotubes. Carbon 50 4945-4952 (2012).

6. Li, M. et al. Nanoelectromechanical resonator arrays for ultrafast, gas-phase chromatographic chemical analysis. Nano Lett. 10, 3899-3903 (2010).

7. Collins, P., Bradley, K., Ishigami, M. \& Zettl, A. Extreme oxygen sensitivity of electronic properties of carbon nanotubes. Science 287, 1801-1804 (2000).

8. Kong, J. et al. Nanotube molecular wires as chemical sensors. Science 287, 622-625 (2000).

9. Jensen, K., Kwanpyo, K. \& Zettl, A. An atomic-resolution nanomechanical mass sensor. Nature Nanotech. 3, 533-537 (2008).

10. Naik, A. K., Hanay, M. S., Hiebert, W. K., Feng, X. L. \& Roukes, M. L. Towards single-molecule nanomechanical mass spectrometry. Nature Nanotech. 4, 445-450 (2009).

11. Mateiu, R., Kuhle, A., Marie, R. \& Boisen, A. Building a multi-walled carbon nanotube-based mass sensor with the atomic force microscope. Ultramicroscopy 105, 233-237 (2005)

12. Peng, H. B., Chang, C. W., Aloni, S., Yuzvinsky, T. D. \& Zettl, A. Ultrahigh Frequency Nanotube Resonators. Phys. Rev. Lett. 97, 087203 (2006).

13. Lassagne, B., Garcia-Sanchez, D., Aguasca, A. \& Bachtold, A. Ultrasensitive Mass Sensing with a Nanotube Electromechanical Resonator. Nano Lett. 8, 3735-3738 (2008)

14. Chiu, H., Hung, P., Postma, H. W. Ch. \& Bockrath, M. Atomic-Scale Mass Sensing Using Carbon Nanotube Resonators. Nano Lett. 8, 4342-4346 (2008).

15. Chaste, J. et al. A nanomechanical mass sensor with yoctogram resolution. Nature Nanotech. 7, 301-304 (2012).

16. Arash, B., Wang, Q. \& Varadan, V. K. Carbon Nanotube-Based Sensors for Detection of Gas Atoms. J. Nanotechnol. Eng. Med. 2, 021010 (2011).

17. Arash, B., Wang, Q. \& Wu, N. Gene Detection With Carbon Nanotubes. J. Nanotechnol. Eng. Med. 3, 020902 (2012).

18. Arash, B., Wang, Q. \& Liew, K. M. Wave propagation in graphene sheets with nonlocal elastic theory via finite element formulation. Comput. Method Appl. M. 223-224, 1-9 (2012)

19. Arash, B. \& Wang, Q. Detection of gas atoms with graphene sheets. Comp. Mater. Sci. 60, 245-249 (2012).

20. Duan, W. H., Gong, K. \& Wang, Q. Controlling the formation of wrinkles in a single layer graphene sheet subjected to in-plane shear. Carbon 49, 3107-3112 (2011)
21. Wang, Q. \& Liew, K. M. Molecular mechanics modeling for properties of carbon nanotubes. J. Appl. Phys. 103, 046103 (2008).

22. Wilson, N. R. \& Macpherson, J. V. Carbon Nanotube Tips for Atomic Force Microscopy. Nat. Nanotechnol. 4, 483-491 (2009).

23. Wei, D. C. \& Liu, Y. Q. The Intramolecular Junctions of Carbon Nanotubes. Adv Mater. 20, 2815-2841 (2008).

24. Weg, C. et al. Fast Active THz Cameras with Ranging Capabilities. J. Infrared Milli. Terahz. Waves 30, 1281-1296 (2009).

25. Gouttenoire, V. et al. Digital and FM Demodulation of a Doubly Clamped SingleWalled Carbon-Nanotube Oscillator: Towards a Nanotube Cell Phone. Small 6 1060-1065 (2010).

26. Song, H. J., Oh, K. H., Shimizu, N., Kukutsu, N. \& Kado, Y. Generation of frequency-modulated subterahertz signal using microwave photonic technique. Opt. Express 18, 15936-15941 (2010).

27. Gurtin, M. E. \& Murdoch, A. I. Surface stress in solids. Int. J. Solids Struct. 14, 431-440 (1978)

28. Eringen, A. C. On differential equations of nonlocal elasticity and solutions of screw dislocation and surface waves. J. Appl. Phys. 54, 4703 (1983).

29. Lei, X., Natsuki, T., Shi, J. \& Ni, Q. Surface effects on the vibrational frequency of double-walled carbon nanotubes using the nonlocal Timoshenko beam model. Compos. Part B-Eng. 43, 64-69 (2012).

30. Rappi, A. K., Casewit, C. J., Colwell, K. S., Goddard, W. A. \& Skid, W. M. UFF, a Full Periodic Table Force Field for Molecular Mechanics and Molecular Dynamics Simulations. J. Am. Chem. Soc. 114, 10024-10035 (1992).

31. Jones, J. E. On the Determination of Molecular Fields-II from the Equation of State of a Gas. Proc. R. Soc. London, Ser. A 106, 463-477 (1924).

32. Polyak, B. T. The conjugate gradient method in extreme problems. URSS Comp. Math. Math. Phys. 9, 94-112 (1969).

33. Andersen, H. C. Molecular dynamics at constant pressure and/or temperature. J. Chem. Phys. 72, 2384-2393 (1980).

\section{Acknowledgement}

This research was undertaken, in part, thanks to funding from the Canada Research Chairs Program (CRC) and the National Science and Engineering Research Council (NSERC).

\section{Author contributions}

B.A. conducted the molecular simulations and theoretical analyses. Q.W. supervised the whole work and contributed to the manuscript preparation. Both authors read and corrected the manuscript before the submission.

\section{Additional information}

Supplementary information accompanies this paper at http://www.nature.com/ scientificreports

Competing financial interests: The authors declare no competing financial interests.

License: This work is licensed under a Creative Commons

Attribution-NonCommercial-NoDerivs 3.0 Unported License. To view a copy of this license, visit http://creativecommons.org/licenses/by-nc-nd/3.0/

How to cite this article: Arash, B. \& Wang, Q. Detection of gas atoms with carbon nanotubes. Sci. Rep. 3, 1782; DOI:10.1038/srep01782 (2013). 